\title{
Vitrificación ovocitaria para posponer fecundidad: experiencia de la Unidad de Medicina Reproductiva de Clínica Monteblanco
}

\author{
Juan Enrique Schwarze M. MSc ${ }^{1,2}$, José Balmaceda R. ${ }^{1}$, Begoña Arguello B. ${ }^{1}$, \\ Claudia Almendra G. ${ }^{1}$, Sonia Villa V. ${ }^{1}$, Ricardo Pommer T. 1,3 \\ ${ }^{1}$ Unidad de Medicina Reproductiva de Clínica Monteblanco; ${ }^{2}$ Departamento Clínico de Obstetricia y Ginecología, \\ Universidad de Santiago; ${ }^{3}$ Instituto de Investigación Materno Infantil de la Universidad de Chile. Santiago, Chile.
}

\section{RESUMEN}

Antecedentes: Múltiples variables médicas y sociales han llevado al desarrollo de técnicas que permitan a la mujer posponer su fertilidad; dentro de ellas la vitrificación ovocitaria surge como una técnica relativamente sencilla y muy promisoria en este campo. Objetivo: Presentar la experiencia del Centro de Medicina Reproductiva de Clínica Monteblanco, en mujeres que han vitrificado sus ovocitos y posteriormente han intentado embarazarse con ellos. Método: Se revisaron todos los casos de vitrificación ovocitaria con posterior intento de embarazo, realizados desde el año 2007 a la fecha. Resultados: Del total de ciclos de vitrificación ovocitaria, 22 mujeres han intentado un embarazo posterior al procedimiento. La mediana de edad al momento de la vitrificación fue de 36 años. En 10 pacientes no hubo transferencia embrionaria, ya fuera por no fecundación (4 casos) o por detención del desarrollo embrionario (6 casos); 12 pacientes fueron transferidas, la mitad de ellas lograron embarazo. Discusión: En este trabajo se presenta la primera serie nacional de casos de vitrificación de óvulos con posterior intento de embarazo; 12 de 20 pacientes lograron ser transferidas y 6 de ellas lograron el embarazo. Las mujeres más jóvenes vitrificaron un mayor número de ovocitos, tuvieron más embriones y tuvieron una mayor posibilidad de lograr embarazo. Conclusión: Pese a que la vitrificación ovocitaria no es una solución definitiva a la postergación de la fertilidad, representa en estos casos una alternativa válida.

\section{PALABRAS CLAVE: Vitrificación ovocitaria, criopreservación ovocitaria, postergación de la fertilidad}

\section{SUMMARY}

Background: The postponement of pregnancy responds to several medical and social reassons. Oocyte vitrification is a relatively simple and promising technique for this purpose. Objective: To illustrate the experience at the Reproductive Medicina Unit of Monteblanco of cases of women who underwent oocyte vitrification and attempted later to get pregnant. Methods: We reviewed all cases of oocyte vitrification with later attempt of pregnancy since 2007. Results: In the studied period, 20 cycles of oocyte vitrification were registered, thereof 22 women attempted a pregnancy after that. Median age at vitrification was 36 years old. Ten patient did not underwent embryo transfer, either by fertilization failure (4 patients) or detention of embryonic development (6 patients). Six of 12 patients that underwent embryo transfer, achieved pregnancy. Discussion: We report the first series of vitrification cases with posterior attempt of pregnancy in Chile; 12 
of 20 women underwent embryo transfer and 6 of them got pregnant. Young women had a more oocytes, embryos and a better chance to achieve pregnancy. Conclusion: Despite the fact that vitrification is not the final solution for today's postponement of fertility, it represents a useful alternative for these cases.

\section{KEY WORDS: Oocyte vitrification, oocyte cryopreservation, postponement of fertility}

\section{INTRODUCCIÓN}

En las últimas décadas las parejas de países desarrollados han variado sustancialmente su comportamiento reproductivo (1). Algunas parejas ven tener hijos como una competencia para otras aspiraciones de la mujer, el hombre y la pareja (2). Al mismo tiempo, muchas de ellas posponen la búsqueda de hijos, hasta conseguir una mejor situación económica. Es así, como la edad media al momento del primer hijo aumentó 2-4 años en muchos países desarrollados en los últimos 20-30 años (3). De este forma, las parejas no sólo desean tener menos hijos que sus predecesores, sino que además desean comenzar a tenerlos más tarde.

Esta situación también se presenta en Chile, aunque con una clara diferencia según el nivel socioeconómico de la mujer. Es así como mujeres de nivel socioeconómico alto, la edad promedio al momento del primer parto es significativamente mayor que en mujeres pertenecientes a segmento socioeconómico bajo y medio bajo. Esto se traduce en un aumento en la frecuencia de embarazos en mujeres mayores, y una disminución en el número de hijos por mujer (4). Lamentablemente, al buscar más tardíamente el embarazo disminuye la posibilidad de conseguirlo por la disminución en la tasa de fecundidad propia del aumento de la edad $(5,6,7)$, y en el caso de lograr embarazo, existe un aumento en la frecuencia de abortos (8).

Una alternativa para paliar esta disminución de las posibilidades de embarazo con el aumento de edad, es el uso de ovocitos donados, que se asocia a una excelente tasa de embarazo independientemente de la edad de la receptora $(9,10)$. Sin embargo, en algunos casos el uso de ovocitos donados no es aceptado por razones éticas o falta de acceso a centros que ofrezcan esta alternativa terapéutica (11).

Últimamente, se ha abierto otra posibilidad con la criopreservación de ovocitos $(12,13)$. En los últimos años la criopreservación de ovocitos ha alcanzado resultados satisfactorios mediante la vitrificación de ellos, publicándose excelentes tasas de sobrevida, de fecundación y embarazo $(14,15)$. Al criopreservar los ovocitos literalmente se "congela en el tiempo" el potencial reproductivo y da la posibilidad de conseguir un embarazo sano cuando la mujer así lo desee. Recientemente publicamos el primer caso de embarazo en Chile tras la transferencia de embriones, producto de la fecundación de ovocitos vitrificados, por lo que comenzamos a ofrecer la posibilidad de este procedimiento a mujeres que desean posponer su fecundidad (16). En el presente reporte presentamos nuestra experiencia en casos de mujeres que han decidido vitrificar ovocitos para postergar fertilidad y luego han vuelto para buscar embarazo.

\section{PACIENTES Y MÉTODO}

Se revisaron todos los casos de vitrificación de ovocitos realizados desde 2007 en la Unidad de Medicina Reproductiva de Clínica Monteblanco, en los que la paciente haya vuelto con el deseo de lograr embarazo.

Hiperestimulación ovárica y culdocentesis. Al segundo o tercer día de menstruación se estimuló con una combinación de FSH recombinante (Puregon, MSD) y urinaria (Menopur, Ferring), en dosis de acuerdo al peso y reserva ovárica de la mujer. Para suprimir la secreción endógena de LH se agregó, desde el sexto día de estimulación, la administración diaria de 0,25 mg de Ganirelix (Orgalutran, MSD), un antagonista de $\mathrm{GnRH}$. El desarrollo folicular fue monitorizado mediante ecografía transvaginal seriada. Una vez que al menos dos folículos alcanzaron un diámetro promedio de $17 \mathrm{~mm}$ se indujo la maduración final de los ovocitos mediante la administración subcutánea de HCG recombinante (Ovidrell, Merck-Serono); 35-37 horas después, se recuperaron los ovocitos mediante aspiración bajo guía ecográfica con anestesia general.

Vitrificación. El protocolo utilizado fue publicado por Kuwayama y cols en el $2005(17,18)$. Según este protocolo, después de 2 horas de cultivo in vitro, los ovocitos fueron denudados de las células de la granulosa con $80 \mathrm{U} / \mathrm{ml}$ de hialurodinasa (SAGE IVF Inc.). Luego fueron expuestos gradualmente a una solución de equilibrio con $7,5 \%$ de EG y $7,5 \%$ de DMSO, seguido por una rápida exposición a la solución de vitrificación, con 15\% de EG, 15\% de 
DMSO y 0,5 M sacarosa. Tras 1 minuto de exposición a esta solución, hasta 2 ovocitos fueron cargados en un contenedor con $0,1 \mu \mathrm{l}$ de volumen de carga y sumergidos rápidamente en nitrógeno líquido. Una vez dentro del nitrógeno, se cubrió con una tapa protectora. Finalmente, los contenedores fueron almacenados en un tanque de almacenamiento con nitrógeno líquido.

Desvitrificación, inseminación y transferencia embrionaria. Los contenedores desvitrificados fueron traspasados desde el tanque de almacenamiento a una caja de poliestireno con nitrógeno líquido, sacando previamente la tapa protectora. Luego, el extremo se sumergió por 1 minuto en una solución de descongelación con sacarosa $1,0 \mathrm{M}$ a $37^{\circ} \mathrm{C}$. A continuación, los ovocitos fueron expuestos por 3 minutos a una solución de sacarosa $0,5 \mathrm{M}$, seguido de 2 pasos por 5 minutos por una solución de lavado. Finalmente, los ovocitos fueron traspasados a una cápsula con medio de cultivo equilibrado e incubados hasta su microinyección, 2 horas después. Los embriones obtenidos fueron cultivados hasta su transferencia, en estadio de ocho células, transvaginal con un catéter ultrasuave, bajo visión ecográfica.

Preparación endometrial. Para preparar el endometrio cuando las pacientes volvieron en busca de embarazo, se administró estradiol oral hasta conseguir un grosor endometrial bajo visión ultrasonográfica de $9 \mathrm{~mm}$. En ese momento se agregó progesterona diaria por vía vaginal. El suplemento exógeno de estradiol y progesterona duró hasta completar el primer trimestre de embarazo, cuando la placenta comienza la síntesis en cantidad adecuada de estos esteroides.

\section{RESULTADOS}

Desde 2007 se han realizado 20 ciclos de vitrificación de ovocitos, 22 de las mujeres han vuelto por busca de embarazo, cuyos datos son incluidos en el presente reporte.

La Tabla I muestra las principales características demográficas del grupo de estudio. La edad al momento de la vitrificación presentó una mediana de 36 años, valor que subió a 37 años al momento de la desvitrificación. La mediana del número de ovocitos criopreservados fue de 20. En 10 casos no se pudo transferir embriones ya sea porque los ovocitos no fecundaron (4 casos) o se detuvo el desarrollo embrionario (6 casos). De los 12 casos con transferencia embrionaria, se embarazó la mitad de las pacientes.
Tabla I

CARACTERÍSTICAS DEMOGRÁFICAS DE LAS PACIENTES QUE DECIDIERON VITRIFICAR OVOCITOS POR RAZONES SOCIALES Y QUE VOLVIERON EN BUSCA DE EMBARAZO

\begin{tabular}{lc}
\hline Características & Mediana (rango) \\
\hline $\begin{array}{l}\text { Edad (años) al momento de la } \\
\text { vitrificación }\end{array}$ & $36(26-45)$ \\
$\begin{array}{l}\text { Edad (años) al momento de la } \\
\text { desvitrificación }\end{array}$ & $37(26-45)$ \\
$\begin{array}{l}\text { Número de ovocitos vitrificados } \\
\text { Número de ovocitos }\end{array}$ & $8(2-23)$ \\
desvitrificados & $4(1-15)$ \\
Número de ovocitos inyectados & $3(0-11)$ \\
Número de ovocitos fecundados & $2(0-9)$ \\
Pacientes con transferencia & $12 / 22$ \\
embrionaria & \\
Pacientes con embarazo & $6 / 12$ \\
\hline
\end{tabular}

En la Tabla II, se muestra las características de las 12 pacientes a las que se les pudo transferir embriones (la mitad de ellas logró embarazo), y 8 pacientes en las que no se logró transferir los embriones. El grupo de mujeres a la que se logró transferir embriones era más joven y vitrificaron más ovocitos. Algo parecido se aprecia dentro del grupo de mujeres a las que se les transfirió embriones, al comparar a las que embarazaron con las que no embarazaron. El grupo de mujeres con embarazo tendió a criopreservar a edades más tempranas y criopreservó más ovocitos.

Las características individuales de las pacientes que lograron embarazo se muestra en la Tabla III. Especialmente ejemplificados son los casos 4 y 5 , que se transfirieron embriones cuando tenían más de 40 años, periodo de la historia reproductiva con una franca declinación en la fecundidad femenina.

\section{DISCUSIÓN}

En esta comunicación presentamos la primera serie nacional de pacientes que criopreservaron óvulos por razones sociales, y que volvieron en busca de embarazo. Encontramos que 12 de 20 pacientes lograron transferir embriones, consiguiendo embarazo en la mitad de ellas. Como era de esperar, en esta serie de casos las mujeres más jóvenes lograron criopreservar más ovocitos y tuvieron más embriones, con lo que aumentó la posibilidad de que al menos uno de ellos lograra implantar. 
Tabla II

\section{CARACTERÍSTICAS DE LAS PACIENTES QUE LOGRARON Y NO LOGRARON EMBARAZO LUEGO DE LA DESVITRIFICACIÓN DE OVOCITOS, VITRIFICADOS INICIALMENTE POR RAZONES SOCIALES}

\begin{tabular}{|c|c|c|c|}
\hline \multirow[t]{2}{*}{ Característica } & \multicolumn{2}{|c|}{ Con transferencia embrionaria } & \multirow{2}{*}{$\begin{array}{c}\text { Sin transferencia } \\
\text { embrionaria } \\
(n=8)\end{array}$} \\
\hline & $\begin{array}{l}\text { Con embarazo } \\
\qquad(n=6)\end{array}$ & $\begin{array}{c}\text { Sin embarazo } \\
(n=6)\end{array}$ & \\
\hline Edad al momento de la vitrificación & $34(26-39)$ & $36(29-42)$ & $39(33-45)$ \\
\hline Edad al momento de la desvitrificación & $35(26-42)$ & $37(29-45)$ & $41(33-45)$ \\
\hline Número de ovocitos vitrificados & $11(2-23)$ & $9(4-10)$ & $6(2-9)$ \\
\hline Número de ovocitos desvitrificados & $10(2-15)$ & $4(3-6)$ & $4(1-7)$ \\
\hline Número de ovocitos inyectados & $8(2-11)$ & $3(3-5)$ & $2(0-6)$ \\
\hline Número de ovocitos fecundados & $6(2-9)$ & $3(2-3)$ & $1(0-3)$ \\
\hline
\end{tabular}

Tabla III

CARACTERÍSTICAS DE LAS PACIENTES QUE LOGRARON EMBARAZO

\begin{tabular}{|c|c|c|c|c|c|c|c|}
\hline Caso & $\begin{array}{c}\text { Edad al } \\
\text { momento } \\
\text { de la } \\
\text { vitrificación }\end{array}$ & $\begin{array}{c}\text { Número de } \\
\text { ovocitos } \\
\text { vitrificados }\end{array}$ & $\begin{array}{c}\text { Edad al } \\
\text { momento de la } \\
\text { desvitrificación }\end{array}$ & Fecundación * & $\begin{array}{l}\text { Número de } \\
\text { embriones } \\
\text { transferidos }\end{array}$ & $\begin{array}{c}\text { Día de la } \\
\text { transferencia } \\
\text { embrionaria }\end{array}$ & $\begin{array}{c}\text { Sacos } \\
\text { gestacionales }\end{array}$ \\
\hline 1 & 26 & 12 & 26 & $2 / 3$ & 2 & 3 & 1 \\
\hline 2 & 30 & 2 & 32 & $2 / 2$ & 2 & 5 & 1 \\
\hline 3 & 37 & 9 & 37 & $7 / 9$ & 2 & 3 & 1 \\
\hline 4 & 39 & 12 & 42 & $9 / 11$ & 2 & 3 & 1 \\
\hline 5 & 37 & 10 & 41 & $8 / 8$ & 2 & 5 & 1 \\
\hline 6 & 29 & 23 & 30 & $5 / 7$ & 2 & 3 & 1 \\
\hline
\end{tabular}

* número de ovocitos fecundados/número de ovocitos inyectados.

Al analizar los casos en que se logró embarazo, llama la atención pacientes que criopreservaron embriones después de los 35 años, y volvieron a buscar embarazo cuando ya tenían más de 40 años, es decir cuando la fecundidad natural ha disminuido ostensiblemente. Si bien estas pacientes no son precisamente infértiles, ya que no se han expuesto a embarazo, la fecundidad propia de la edad es baja, por lo que probablemente la vitrificación ovocitaria permitió prolongar su fecundidad (19).

La criopreservación de ovocitos es una especie de cuenta de ahorro para fecundidad futura, pero al igual que cualquier otra técnica médica en general y de reproducción asistida en particular, no es completamente eficiente (8). Por lo tanto, no es una solución definitiva para el problema de postergación de la maternidad. Otras medidas políticas y sociales deben suplir este problema. Sin embargo, estudios realiza- dos en países industrializados (20), concluyen que una significativa proporción de la población femenina consideraría la preservación de su fecundidad por medio de la vitrificación de ovocitos.

Si bien la recomendación es que el proceso se realice antes de los 35 años, nuestra serie demuestra que si es posible conseguir embarazo cuando la vitrificación de ovocitos se hace después de los 39 años, lo que concuerda con la experiencia de otros autores (21). En parte esto se podría explicar en que estas mujeres no son infértiles per sé, ya que no han estado expuestas al riesgo de embarazo, por lo que los paradigmas a los que estamos habituados en reproducción asistida, pueden que no sean aplicables en estos casos en particular. Confiamos en que nuevas series publicadas por otros centros nos permitan obtener más información para orientar a nuestras pacientes. 


\section{REFERENCIAS}

1. Baird DT, Collins J, Egozcue J, Evers LH, Gianaroli L, Leridon $\mathrm{H}$, et al. Fertility and ageing. Hum Reprod Update 2005;11:261-76.

2. Verhoef R, Van De Kaa DJ. Population registers and population statistics. Popul Index 1987;53:633-42.

3. Sobotka T, Skirbekk V, Philipov D. Economic recession and fertility in the developed world. Popul Dev Rev 2011;37:267-306.

4. Fuentes A, Jesam C, Devoto L, Angarita B, Galleguillos A, Torres A, Mackenna A. [Association between motherhood postponement and socioeconomic status]. Rev Med Chil 2010;138:1240-5.

5. Gurtcheff SE, Klein NA. Diminished ovarian reserve and infertility. Clin Obstet Gynecol 2011;54:666-74.

6. Younis JS. Ovarian aging: latest thoughts on assessment and management. Curr Opin Obstet Gynecol 2011;23:427-34.

7. Liu K, Case A. Advanced reproductive age and fertility. J Obstet Gynaecol Can 2011;33:1165-75.

8. Lockwood GM. Social egg freezing: the prospect of reproductive 'immortality' or a dangerous delusion? Reprod Biomed Online 2011;23:334-40.

9. Borini A, Suriano R, Barberi M, Dal Prato L, Bulletti C. Oocyte donation programs: strategy for improving results. Ann N Y Acad Sci 2011;1221:27-31.

10. Oyesanya OA, Olufowobi O, Ross W, Sharif K, Afnan M. Prognosis of oocyte donation cycles: a prospective comparison of the in vitro fertilization-embryo transfer cycles of recipients who used shared oocytes versus those who used altruistic donors. Fertil Steril 2009;92:930-6.

11. Klein JU, Sauer MV. Ethics in egg donation: past, present, and future. Semin Reprod Med 2010;28:322-8.

12. Chang CC, Shapiro DB, Bernal DP, Wright G, Kort $H I$, Nagy ZP. Human oocyte vitrification: in-vivo and in-vitro maturation outcomes. Reprod Biomed Online 2008;17:684-8.

13. Cobo A, Bellver J, Domingo J, Pérez S, Crespo J, Pellicer A, Remohí J. New options in assisted reproduction technology: the Cryotop method of oocyte vitrification. Reprod Biomed Online 2008;17:68-72.

14. Molloy D, Hall BA, Ilbery M, Irving J, Harrison KL. Oocyte freezing: timely reproductive insurance? Med J Aust 2009;190:247-9.

15. Rienzi L, Cobo A, Paffoni A, Scarduelli C, Capalbo A, Vajta $G$, et al. Consistent and predictable delivery rates after oocyte vitrification: an observational longitudinal cohort multicentric study. Hum Reprod 2012;27:1606-12.

16. Pommer R, Jeria F, Arguello B, Villa S, Balmaceda J, Schwarze E. [Live birth after the transfer of embryos obtained by fertilization of vitrified oocytes: Report of one case]. Rev Med Chil 2011;139:920-3.

17. Kuwayama M, Vajta G, Kato O, Leibo SP. Highly efficient vitrification method for cryopreservation of human oocytes. Reprod Biomed Online 2005;11:300-8.

18. Kuwayama M, Vajta G, leda S, Kato O. Comparison of open and closed methods for vitrification of human embryos and the elimination of potential contamination. Reprod Biomed Online 2005;11:608-14.

19. Zegers-Hochschild F, Adamson GD, de Mouzon J, Ishihara O, Mansour R, Nygren K, et al. International Committee for Monitoring Assisted Reproductive Technology (ICMART) and the World Health Organization (WHO) revised glossary of ART terminology, 2009. Fertil Steril 2009;92:1520-4.

20. Stoop D, Nekkebroeck J, Devroey P. A survey on the intentions and attitudes towards oocyte cryopreservation for non-medical reasons among women of reproductive age. Hum Reprod 2011;26:655-61.

21. Mertes $H$, Pennings G. Social egg freezing: for better, not for worse. Reprod Biomed Online 2011;23:824-9. 